OPEN ACCESS

Edited by:

Peter Korsten

University Medical Center

Göttingen, Germany

Reviewed by:

Gunnar N. Hillerdal,

Karolinska University

Hospital, Sweden

Viktor Korendovych

University Medical Center

Göttingen, Germany

*Correspondence:

Antonio De Cinque

antdecinque@hotmail.it

†These authors have contributed equally to this work and share last authorship

Specialty section:

This article was submitted to

Pulmonary Medicine,

a section of the journal

Frontiers in Medicine

Received: 25 September 2020 Accepted: 18 December 2020 Published: 27 January 2021

Citation:

De Cinque A, Corcioni B, Rossi MS, Franceschelli A, Colombo F, Golfieri R, Renzulli M and Gaudiano C (2021) Case Report: Testicular Sarcoidosis: The Diagnostic Role of Contrast-Enhanced Ultrasound and Review of the Literature. Front. Med. 7:610384. doi: 10.3389/fmed.2020.610384

\section{Case Report: Testicular Sarcoidosis: The Diagnostic Role of Contrast-Enhanced Ultrasound and Review of the Literature}

\author{
Antonio De Cinque ${ }^{1 *}$, Beniamino Corcioni ${ }^{1}$, Martina Sofia Rossi ${ }^{2}$, \\ Alessandro Franceschelli ${ }^{2,3}$, Fulvio Colombo ${ }^{2,3}$, Rita Golfieri ${ }^{1}$, Matteo Renzulli ${ }^{1+}$ and \\ Caterina Gaudiano ${ }^{1 \dagger}$ \\ ${ }^{1}$ Department of Radiology, Azienda Ospedaliero-Universitaria di Bologna, Bologna, Italy, ${ }^{2}$ Department of Urology, Azienda \\ Ospedaliero-Universitaria di Bologna, Bologna, Italy, ${ }^{3}$ Andrology-Unit, Azienda Ospedaliero-Universitaria di Bologna, \\ Bologna, Italy
}

Sarcoidosis is a multisystemic disease histologically characterized by non-caseating epithelioid granulomas and multinucleated giant cells; the etiology is still uncertain, and likely related to a complex interplay between environmental and genetic factors. The genitourinary system is affected in fewer than $0.2 \%$ of all clinically diagnosed cases of sarcoidosis and in 5\% of those identified in autopsy studies. In this report, we describe a case of a 42-year-old male with one hypoechoic lesion per testis on B-mode evaluation; contrast-enhanced ultrasound (CEUS) on both lesions was carried out. During the early phase, the masses showed a hypovascular appearance as compared to the surrounding testicular tissue, maintaining the hypo-enhancement in the late phase. Tissue biopsy for pathological evaluation confirmed testicular sarcoid involvement, showing non-caseating granulomas. Allowing visualization of testicular microvascularisation, CEUS may play an important role in excluding malignancy, avoiding unnecessary aggressive treatment for benign conditions, such as sarcoidosis. A review of the literature of reported cases since 2004 of sarcoidosis involving the testis is also included.

Keywords: andrology, urology, sarcoidosis, ultrasonography, contrast media

\section{INTRODUCTION}

Sarcoidosis is a multisystemic disease that usually affects patients in the fifth decade of their life with a variable incidence rate depending on countries and ethnic group (1). A recent study from the Mayo Clinic reported an incidence rate of 11 per 100,000 people/year among a cohort mostly composed of white people (2) while another study from the United States estimated an incidence rate of 8.1 per 100,000 people/year in Caucasians, 17.8 in African Americans, 4.3 in Hispanics and 3.2 in Asians (3). In Europe, an incidence of 11.5 per 100,000 people/year has been reported in Sweden (4) and of 5.0 in the UK (5). The incidence rate of sarcoidosis is increasing over time in developed countries, such as Korea, together with an increase in the age of diagnosis probably due to population aging (6).

Sarcoidosis is histologically characterized by non-caseating epithelioid granulomas and multinucleated giant cells. Its etiology is still uncertain, and likely related to a complex relationship between environmental and genetic factors (7-9). 
Sarcoidosis is characterized by bilateral chest hilar lymphadenopathy and/or reticulonodular pulmonary infiltrates in the vast majority of cases (>90\%). However, this systemic pathology can involve any organ (10): among them the genitourinary system is involved in $5 \%$ of cases identified in autoptic studies and in fewer than $0.2 \%$ of clinically diagnosed cases. According to a review published in 2004, the male genitourinary organs most frequently involved by sarcoidosis are the epididymis (73\%), the testis (47\%), the spermatic cord $(8 \%)$ and the prostate $(3 \%)$ (11). In particular, the total number of testicular sarcoidosis accounted for 28 cases in 2004. We performed a new review of the literature, finding additional cases involving the testis associated with histologically proven diagnosis.

Testicular sarcoid presentation can vary from testicular swelling to painless or painful unilateral or bilateral masses (12, 13). Especially in cases of painless unilateral mass, the differential diagnosis is complex and difficult, ranging from testicular malignancies to infections, and could result in diagnostic errors leading to inappropriate and unnecessary treatments, such as the orchiectomy (13).

Usually the achievement of a correct diagnosis of sarcoidosis with genitourinary and in particular testicular involvement relies on the association between clinical and imaging findings (14). The most utilized imaging techniques for this issue are Ultrasound (US), Magnetic Resonance Imaging (MRI) and Positron Emission Tomography-Computed Tomography (PET-CT).

New and non-invasive imaging techniques, such as contrastenhanced US (CEUS), have recently been refined and adopted in many guidelines $(15,16)$. However, to the best of our knowledge, this is the first report in the English literature to explore in detail the potentiality of CEUS in a case of histologically proven testicular sarcoidosis.

A very rare case of testicular sarcoidosis is herein reported together with a detailed review of the literature highlighting the role of CEUS to confidently achieve the final diagnosis excluding other possible differential diagnoses, such as testicular tumor masses.

\section{CASE REPORT}

A 42-year-old Caucasian male was admitted to our Hospital referring a 4-month history of gradually increasing upper left quadrant pain. His medical history included bladder neck sclerosis, cholecystectomy, diabetes insipidus, hypogonadotropic hypogonadism, allergic asthma, and a smoking history until 10 months before this admission. The medical examination did not show any relevant features and, therefore, the subsequent diagnostic work-up continued with abdominal US.

Abdominal US showed multiple hypoechoic splenic and hepatic lesions ranging from 5 to $27 \mathrm{~mm}$ and, therefore, the patient underwent total body CT scan for a correct lesions characterization and staging. CT scan demonstrated bilateral hilar lymphadenopathies in the chest, pulmonary perilymphatic micronodules, enlarged retroperitoneal lymph nodes, and confirmed the hepatic and splenic lesions (Figures 1A,B). The first diagnostic hypothesis was the lymphoma and a ${ }^{18} \mathrm{~F}$ Fludeoxyglucose $\left({ }^{18} \mathrm{~F}-\mathrm{FDG}\right) \mathrm{PET} / \mathrm{CT}$ was performed, showing increased ${ }^{18}$ F-FDG uptake (standardized uptake value (SUV) $\max =17$ at the chest hilar lymphadenopathies level) in all the lesions revealed on CT (Figure 1C). Moreover, a focal uptake was detected in left testis (Figure 1D). A brain MRI excluded central nervous system involvement. Lymphopenia was the only abnormal blood cell count value. Alpha-fetoprotein (AFP), Human chorionic gonadotropin (HCG), Aspartate aminotransferase (AST), Alkaline phosphatase (ALP) were within their normal ranges, while alanine aminotransferase (ALT) (55 U/L), gamma-glutamyl transferase (GGT) (60 $\mathrm{U} / \mathrm{L}), \mathrm{C}$-reactive protein (CRP) $(0.82 \mathrm{mg} / \mathrm{dL})$ and Erythrocyte sedimentation rate (ESR) $(20 \mathrm{~mm})$ were mildly elevated. Moreover, Angiotensin Converting Enzyme (ACE) levels were elevated $(95 \mathrm{U} / \mathrm{L})$. He had no history or signs of tuberculosis (QuantiFERON ${ }^{\circledR}$-TB Gold Plus test was negative).

These findings raised the suspicion of lymphoma or sarcoidosis. An endobronchial ultrasound-guided transbronchial needle aspiration (EBUS-TBNA) was performed on the chest hilar lymphadenopathies, showing non-caseating granulomas, consistent with sarcoidosis. Stains for acid-fast bacilli and polymerase chain reaction (PCR) in specimens were negative. The final diagnosis was sarcoidosis with multiorgan involvement. A testicular US evaluation was carried out using a CanonToshiba Aplio $500^{\mathrm{TM}}$ (Otawara, Kanto, Japan) with a high frequency (4-14 MHz) linear transducer. The US demonstrated, using the B-mode evaluation, a hypoechoic lesion of $20 \mathrm{~mm}$ with ill-defined margins in the left testis (Figure 2A) corresponding to the lesion identified on PET-CT; moreover, differently from the latter technique, the US identified a smaller and wellshaped hypoechoic lesion also in the right testis $(6 \mathrm{~mm})$. The Color Doppler demonstrated the presence of vascular flow within the lesions (Figure 2B). Therefore, it was decided to perform CEUS, conducted with the administration of $4.8 \mathrm{ml}$ of second-generation contrast media (SonoVue ${ }^{\mathrm{TM}}$, Bracco, Milano, Italy) followed by $10 \mathrm{~mL}$ of $0.9 \%$ saline solution. Both the testicular lesions demonstrated the same pattern on CEUS. In particular, during the arterial phase, the masses showed a hypovascular appearance as compared to the surrounding testicular tissue (Figure 2C), maintaining the hypo-enhancement in the late phase. This CEUS pattern was not typical of the most frequent testicular tumors such as seminomas, which usually are arterialized appearing hyperechoic on CEUS (17). Finally, the imaging diagnosis was testicular sarcoidosis, based principally on bilateral involvement of testes on CEUS and on the CEUS pattern (hypoenhancement). Testicular sarcoid involvement was confirmed by surgical biopsy of both testicular masses that demonstrated non-caseating granulomas.

The patient was treated with corticosteroids and then with a second-line therapy (methotrexate), thus achieving a reduction of the SUVmax in all of the sarcoid lesions (SUVmax $=2.6$ at the testicular level) at 6- and 12-month PET/CT follow-up.

According to our review (Table 1), from 2004 (11) until now we have identified 20 cases (including the present one) of testicular sarcoidosis. Finally, to date, the total number of 


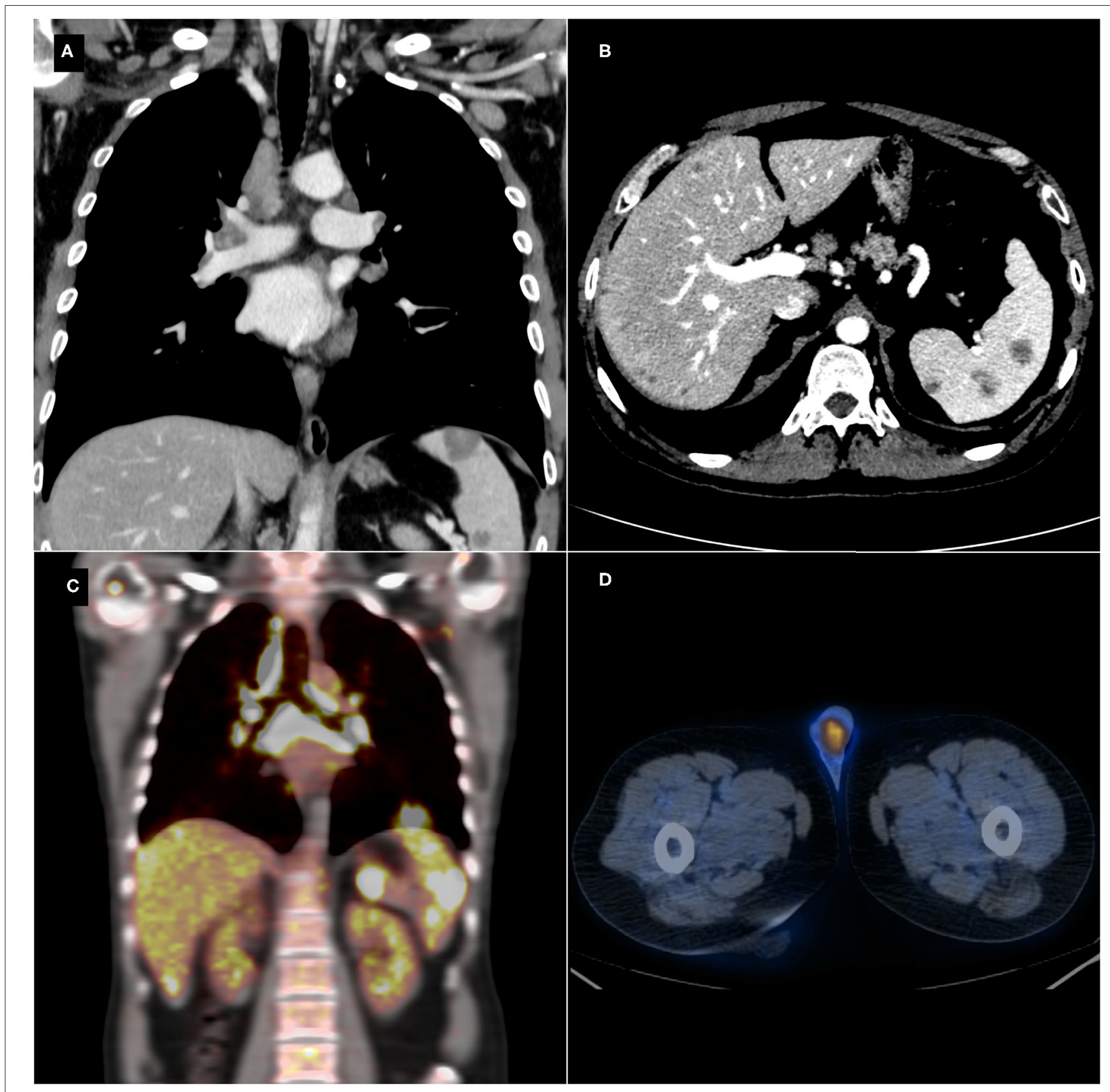

FIGURE 1 | Thoraco-abdominal contrast-enhanced computed tomography (CT) scan showing hilar and mediastinal lymphadenopathies (A), enlarged retroperitoneal lymph nodes, and multiple hepatic and splenic hypodense lesions (B). Positron emission tomography-computed tomography (PET/CT) detecting an increased focal ${ }^{18} \mathrm{~F}$-Fludeoxyglucose $\left({ }^{18} \mathrm{~F}-\mathrm{FDG}\right)$ uptake in the mediastinal and abdominal lesions (C) and in the left testis (D).

histologically proven testicular sarcoidosis published in literature account for 48 cases.

\section{DISCUSSION}

Testicular masses may have many differential diagnoses including malignancies and, very rarely, benign processes such as traumatic or infective/inflammatory lesions including sarcoidosis which incidence is increasing $(6,35)$. US has a pivotal role in investigating testicular lesions since it is extremely accurate in the detection of the masses, even very small ones, due to its high spatial resolution (35). Moreover, the US often represents the sole imaging technique needed prior to surgery, although the recognition of benign entities may be challenging (36). The most common US findings in testicular sarcoidosis are multifocal small hypoechoic 

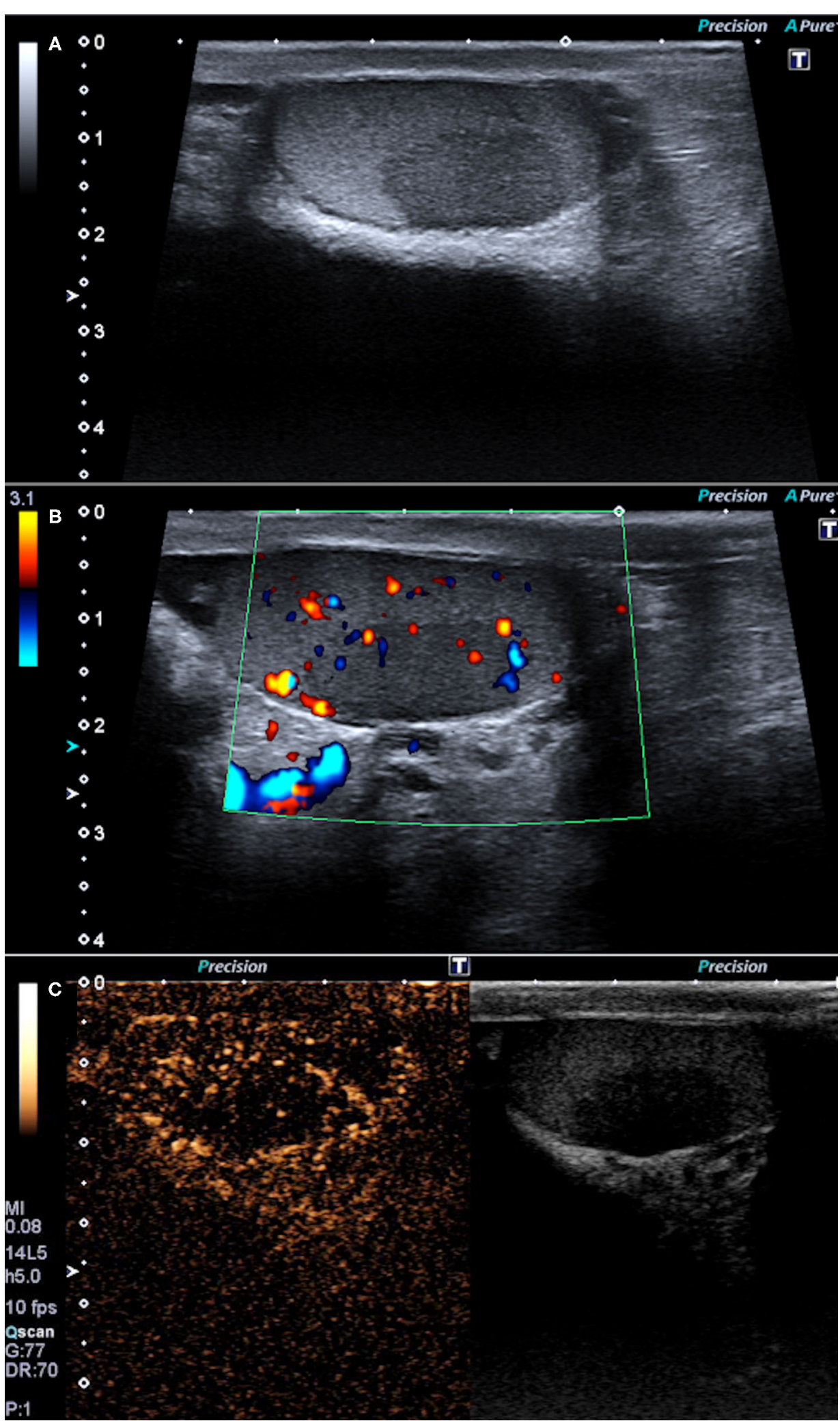

FIGURE 2 | B-mode ultrasonography showing one hypoechoic lesion in the left testis with ill-defined margins (A) and some Color-Doppler flow (B). Contrast-enhanced ultrasonography showing the hypovascular appearance of the lesions as compared to the surrounding testicular tissue (C) 
TABLE 1 | Histologically proven cases of sarcoidosis involving the testis reported in the English literature from 2004 to 2020 (including the present case).

\begin{tabular}{llll}
\hline References & Imaging & Uni- or Bilateral & Orchiectomy \\
\hline Rees et al. (18) & US & Bilateral & No \\
Rehman et al. (19) & US & Bilateral & No \\
Massarweh et al. & US & Bilateral & No \\
(12) & & & \\
Thuret et al. (20) & US & Unilateral (R) & Yes \\
Real et al. (21) & US & Bilateral & Yes \\
Gupta and Senadhi & US & Unilateral (R) & Yes \\
(13) & & & \\
Kim et al. (22) & US & Unilateral (R) & No \\
Paknejad et al. (23) & US & Nilateral & No \\
Kovac et al. (24) & N/A & No \\
Esnakula et al. (25) & US & Unilateral (R) & Yes \\
Joel et al. (26) & US & Unilateral (L) & Yes \\
Patel et al. (27) & US & Bilateral & No \\
Knox et al. (28) & N/A & Bilateral & Yes \\
Chierigo et al. (29) & US & Bilateral & No \\
Babst et al. (30) & US & Bilateral & No \\
Konishi et al. (31) & US, CT & Bilateral & No \\
Hamitouche et al. & US & Bilateral & No \\
(32) & & & \\
Kimura et al. (33) & US, MRI, Gallium-67 & Bilateral & No \\
Parida et al. (34) & Scintigraphy & & \\
This study & FDG-PET/CT, US, & Bilateral & No \\
& CEUS & & \\
\hline
\end{tabular}

US, Ultrasound; CT, Computed Tomography; MRI, Magnetic Resonance Imaging; FDG-PET/CT, Fludeoxyglucose-Positron Emission Tomography/Computed Tomography; CEUS, Contrast-enhanced ultrasound; $R$, right; L, left; N/A, not available.

lesions which can range from a few millimeters to a few centimeters, with well-circumscribed or ill-defined margins. Bilateral unifocal lesions, as in the present case, are less common (37).

However, a question remains unsolved: what is the correct diagnosis of the left testicular lesion? In fact, the testicular sarcoid involvement is very rare, and an association between testicular cancer and sarcoidosis has been reported (38). Moreover, the ${ }^{18}$ F-FDG uptake was detected only in the left testis (not in both testes) and the max SUV of the left testicular lesion (SUVmax $=11.6$ ) was different from the sarcoid lesions involving the other organs. All these findings could not allow the exclusion of a testicular tumor.

In our case, on Color Doppler US some areas of vascularity within the lesion were detected. Usually, the color flow detectable in primary tumors of the testis using this imaging technique is higher than that of non-neoplastic lesions. However, some malignant lesions of the testis could lack internal vascularity on Color Doppler, such as burned-out testis tumors, being difficult to characterize with respect to non-neoplastic lesions (39). Therefore, the sole use of Color-Doppler US was not sufficient to exclude the possible
TABLE 2 | Differential diagnosis of testicular sarcoidosis with the most frequent testicular lesions on B-Mode ultrasound and contrast-enhanced ultrasound.

\begin{tabular}{lll}
\hline & B-Mode US & CEUS \\
\hline Seminoma & Hypoechoic & Fast wash-in and rapid washout \\
Leydigioma & Hypoechoic & Wash-in and delayed washout \\
Sarcoidosis & Hypoechoic & Hypo-enhancement \\
Lymphoma & Hypoechoic & Fast wash-in and rapid washout \\
& & ("straight vessel pattern") \\
Testicular adrenal & Hypoechoic & Fast wash-in and delayed \\
rest tumors & & washout
\end{tabular}

* "Straight vessel pattern" (or "nonbranching linear pattern") of increased vascularity represents a parallel arrangement of testicular small vessels that reflects the interstitial growth pattern of lymphoma, which preserves the vascular architecture of the testis. US, Ultrasound; CEUS, Contrast-enhanced ultrasound.

diagnosis of testicular tumor lesion. Furthermore, ${ }^{18}$ F-FDGPET scan has high sensitivity in detecting lesions having an increased glucose uptake, but it is unable to differentiate an inflammatory process such as sarcoidosis from malignancy (40). Furthermore, in our case, the right testicular lesion did not demonstrate ${ }^{18}$ F-FDG uptake probably due the small lesion dimension under the resolution power of this technique.

In the present case, the testicular unifocal lesions did not demonstrate hyperenhancement on CEUS and, moreover, the lesions were bilateral. The CEUS hyperenhancement of a testicular lesion has a positive predictive value of $97 \%$ for neoplasia and, although its presence alone is not specific enough to establish an unequivocal diagnosis, it is suggestive for a neoplastic testicular lesion, including malignancy (41). Moreover, the most common testicular malignancies in the same age of the patient described in this case are the testicular germ cell tumors that are bilateral in only $2 \%$ of cases (42).

The sarcoid testicular masses could pose problems of differential diagnosis with other bilateral testicular lesions that appear hypoechoic on B-mode US (Table 2), such as Seminoma, Leydigiomas, lymphomas, and testicular adrenal rest tumors. Seminomas usually demonstrate rapid wash-in coupled with rapid washout on CEUS (17). Leydigiomas show hyperenhancement as compared to the surrounding testicular parenchyma on CEUS, with delayed wash-out (17) and therefore the differential diagnosis with sarcoid testicular masses appear easy to perform. Lymphomas, along with leukemia, show marked CEUS hypervascularization (visible also with Color Doppler), a "nonbranching linear pattern" (known also as "straight vessel pattern") and a rapid filling time $(43,44)$, differently from sarcoid testicular masses. Testicular adrenal rest tumors demonstrate high hypervascularization as compared to the sarcoid lesions; moreover, these rare lesions arise in younger patients with congenital adrenal hyperplasia (45). Different approach could be have in case of oncological patients, in whom metastases (the most common are from prostate carcinoma, melanoma, colon and kidney cancer) are rare 
but could not be excluded especially in cases of advanced malignancy (46).

A possible limitation of our case was the absence of MRI evaluation. However, MRI is not able to identify a specific pattern for sarcoidosis (47), and therefore it was not performed after CEUS in our case.

In conclusion, CEUS could play an important role in the evaluation of testicular lesions, and in particular of benign lesions, such as sarcoidosis. Due to the rising incidence rate of this disease and the reported association with testicular cancer, the need to establish a correct differential diagnosis will likely increase over time. CEUS could allow to achieve a correct diagnosis of testicular lesions, due to its ability in identifying very small testis lesions, such as sarcoidosis, coupled with its high accuracy in excluding malignancies, thus avoiding aggressive and potentially unnecessary maneuvers, such as biopsy for benign conditions.

\section{REFERENCES}

1. Arkema EV, Cozier YC. Epidemiology of sarcoidosis: current findings and future directions. Ther Adv Chronic Dis. (2018) 9:227-40. doi: 10.1177/2040622318790197

2. Ungprasert $\mathrm{P}$, Crowson CS, Matteson EL. Influence of gender on epidemiology and clinical manifestations of sarcoidosis: a populationbased retrospective cohort study 1976-2013. Lung. (2017) 195:87-91. doi: 10.1007/s00408-016-9952-6

3. Baughman RP, Field S, Costabel U, Crystal RG, Culver DA, Drent M, et al. Sarcoidosis in America. Analysis based on health care use. Ann Am Thorac Soc. (2016) 13:1244-52. doi: 10.1513/AnnalsATS.201511-760OC

4. Arkema EV, Grunewald J, Kullberg S, Eklund A, Askling J. Sarcoidosis incidence and prevalence: a nationwide register-based assessment in Sweden. Eur Respir J. (2016) 48:1690-9. doi: 10.1183/13993003.00477-2016

5. Gribbin J, Hubbard RB, Le Jeune I, Smith CJ, West J, Tata LJ. Incidence and mortality of idiopathic pulmonary fibrosis and sarcoidosis in the UK. Thorax. (2006) 61:980-5. doi: 10.1136/thx.2006.062836

6. Yoon HY, Kim HM, Kim YJ, Song JW. Prevalence and incidence of sarcoidosis in Korea: a nationwide population-based study. Respir Res. (2018) 19:158. doi: 10.1186/s12931-018-0871-3

7. Gerke AK, Hunninghake G. The immunology of sarcoidosis. Clin Chest Med. (2008) 29:379-90. doi: 10.1016/j.ccm.2008.03.014

8. Müller-Quernheim J, Schürmann M, Hofmann S, Gaede KI, Fischer A, Prasse A, et al. Genetics of sarcoidosis. Clin Chest Med. (2008) 29:391-414, viii. doi: 10.1016/j.ccm.2008.03.007

9. Newman LS, Rose CS, Bresnitz EA, Rossman MD, Barnard J, Frederick $\mathrm{M}$, et al. A case control etiologic study of sarcoidosis: environmental and occupational risk factors. Am J Respir Crit Care Med. (2004) 170:1324-30. doi: 10.1164/rccm.200402-249OC

10. Baughman RP, Teirstein AS, Judson MA, Rossman MD, Yeager H Jr, Bresnitz EA, et al. Clinical characteristics of patients in a case control study of sarcoidosis. Am J Respir Crit Care Med. (2001) 164:1885-9. doi: 10.1164/ajrccm.164.10.2104046

11. Kodama K, Hasegawa T, Egawa M, Tomosugi N, Mukai A, Namiki M. Bilateral epididymal sarcoidosis presenting without radiographic evidence of intrathoracic lesion: review of sarcoidosis involving the male reproductive tract. Int J Urol. (2004) 11:345-8. doi: 10.1111/j.1442-2042.2004.00783.x

12. Massarweh NN, Bhalani VK, Shaw KK, Crawford B, Lang E, Davis R. Testicular presentation of sarcoidosis and organ preservation: case report and review of management strategies. Urology. (2006) 67:200. doi: 10.1016/j.urology.2005.08.011

\section{DATA AVAILABILITY STATEMENT}

The original contributions presented in the study are included in the article/supplementary material, further inquiries can be directed to the corresponding author/s.

\section{ETHICS STATEMENT}

Ethical review and approval was not required for the study on human participants in accordance with the local legislation and institutional requirements. The patient has given his written informed consent to publish the case (including publication of images).

\section{AUTHOR CONTRIBUTIONS}

All authors listed have made a substantial, direct and intellectual contribution to the work, and approved it for publication.

13. Gupta R, Senadhi V. A diagnostic dilemma: metastatic testicular cancer and systemic sarcoidosis - a review of the literature. Case Rep Oncol. (2011) 4:118-24. doi: 10.1159/000324184

14. Stewart VR, Sidhu PS. The testis: the unusual, the rare and the bizarre. Clin Radiol. (2007) 62:289-302. doi: 10.1016/j.crad.2006.10.005

15. Sidhu PS, Cantisani V, Dietrich CF, Gilja OH, Saftoiu A, Bartels E, et al. The EFSUMB guidelines and recommendations for the clinical practice of contrast-enhanced ultrasound (CEUS) in non-hepatic applications: update 2017 (long version). Ultraschall Med. (2018) 39:e2-44. doi: 10.1055/a-0586-1107

16. European Association for the Study of the Liver. EASL Clinical Practice Guidelines: Management of hepatocellular carcinoma. J Hepatol. (2018) 69:182-236. doi: 10.1016/j.jhep.2018.03.019

17. Isidori AM, Pozza C, Gianfrilli D, Giannetta E, Lemma A, Pofi R, et al. Differential diagnosis of nonpalpable testicular lesions: qualitative and quantitative contrast-enhanced US of benign and malignant testicular tumors. Radiology. (2014) 273:606-18. doi: 10.1148/radiol.14132718

18. Rees DA, Dodds AL, Rathbone N, Davies JS, Scanlon MF. Azoospermia in testicular sarcoidosis is an indication for corticosteroid therapy. Fertil Steril. (2004) 82:1672-4. doi: 10.1016/j.fertnstert.2004.07.950

19. Rehman J, Rizkala ER, Chughtai B, Khan SA. Hypoechoic testicular mass: a case of testicular and epididymal sarcoidosis. Urology. (2005) 66:657. doi: 10.1016/j.urology.2005.03.008

20. Thuret R, Cariou G, Aerts J, Cochand-Priollet B. Testicular sarcoidosis with elevated levels of cancer-associated markers. J Clin Oncol. (2008) 26:6007-8. doi: 10.1200/JCO.2008.17.9861

21. Real V, de Loyola GL, Zanoni PE, Real LF. Testicular sarcoidosis: a diagnosis to be considered. Rev Col Bras Cir. (2011) 38:145-6. doi: 10.1590/S0100-69912011000200015

22. Kim YB, Chung YG, Kim SJ, Kim SJ, Ahn HS, Joo HJ, et al. Extensive systemic sarcoidosis with testicular involvement mimicking metastatic testicular cancer. Korean J Urol. (2011) 52:295-7. doi: 10.4111/kju.2011.52.4.295

23. Paknejad O, Gilani MA, Khoshchehreh M. Testicular masses in a man with a plausible sarcoidosis. Indian J Urol. (2011) 27:269-71. doi: 10.4103/0970-1591.82848

24. Kovac JR, Flood D, Mullen JB, Fischer MA. Diagnosis and treatment of azoospermia resulting from testicular sarcoidosis. J Androl. (2012) 33:162-6. doi: 10.2164/jandrol.110.012534

25. Esnakula AK, Coleman P, Ahaghotu CA, Naab TJ. Scrotal mass and unilateral lung masses with pleural effusion mimicking metastatic testicular malignancy: an unusual presentation of sarcoidosis. BMJ Case Rep. (2013) 2013:bcr2012008658. doi: 10.1136/bcr-2013-008658 
26. Joel J, Thomas J, Gill K, Biyani CS. Testicular sarcoidosis masquerading as testicular carcinoma. Cent European J Urol. (2014) 67:261-3. doi: 10.5173/ceju.2014.03.art10

27. Patel H, Shaaban H, Kumar A, Modi T, Maroules M. A rare case report of bilateral testicular masses as an initial manifestation of systemic sarcoidosis. Urol Ann. (2015) 7:378-9. doi: 10.4103/0974-7796.152046

28. Knox A, Black N, Agbaje I. Pretesticular and testicular effects of systemic sarcoidosis: a case report. J Reprod Med. (2017) 62:204-6.

29. Chierigo F, Alnajjar HM, Haider A, Walkden M, Shaikh T, Muneer A. Testicular pain as an atypical presentation of sarcoidosis. Ann R Coll Surg Engl. (2019) 101:e99-101. doi: 10.1308/rcsann.2019.0015

30. Babst C, Piller A, Boesch J, Schmid HP. Testicular sarcoidosis. Urol Case Rep. (2018) 17:109-10. doi: 10.1016/j.eucr.2018.01.021

31. Konishi S, Hatakeyama S, Yoneyama T, Yoneyama T, Hashimoto Y, Ohyama C. Bilateral scrotal mass mimicking testicular cancer: an unusual presentation of sarcoidosis. Int J Urol. (2019) 26:1079-81. doi: 10.1111/iju.14089

32. Hamitouche F, Lacoste M, Korenbaum C, Galland J. Diffuse lesions. Rev Med Interne. (2019) 40:476-7. doi: 10.1016/j.revmed.2018.09.005

33. Kimura S, Momozono K, Shimamatsu K, Noguchi M. Testicular sarcoidosis with bilateral scrotal swelling. IJU Case Rep. (2019) 3:12-4. doi: 10.1002/iju5.12124

34. Parida GK, Kumar A, Mitra S, Suman A, Muthu GS. Rare case of testicular sarcoidosis detected on FDG PET/CT in a patient with PUO. Clin Nucl Med. (2020) 45:e368-9. doi: 10.1097/RLU.0000000000003120

35. Appelbaum L, Gaitini D, Dogra VS. Scrotal ultrasound in adults. Semin Ultrasound CT MR. (2013) 34:257-73. doi: 10.1053/j.sult.2013.01.008

36. Bertolotto $M$, Muça $M$, Currò F, Bucci S, Rocher L, Cova MA. Multiparametric US for scrotal diseases. Abdom Radiol. (2018) 43:899-917. doi: 10.1007/s00261-018-1510-7

37. Eraso CE, Vrachliotis TG, Cunningham JJ. Sonographic findings in testicular sarcoidosis simulating malignant nodule. J Clin Ultrasound. (1999) 27:81-3. doi: 10.1002/(SICI)1097-0096(199902)27:2 <81::AID-JCU6>3.0.CO;2-N

38. Paparel P, Devonec M, Perrin P, Ruffion A, Decaussin-Petrucci M, Akin O, et al. Association between sarcoidosis and testicular carcinoma: a diagnostic pitfall. Sarcoidosis Vasc Diffuse Lung Dis. (2007) 24:95-101.

39. Miacola C, Colamonico O, Bettocchi C, Ricapito V, Palazzo S, Campagna $M$, et al. Burned-out in a mixed germ cell tumor of the testis: the problem of pT0. Case report. Arch Ital Urol Androl. (2014) 86:389-90. doi: 10.4081/aiua.2014.4.389

40. Akaike G, Itani M, Shah H, Ahuja J, Yilmaz Gunes B, Assaker R, et al. $\mathrm{PET} / \mathrm{CT}$ in the diagnosis andworkup of sarcoidosis: focus on atypical manifestations. Radiographics. (2018) 38:1536-49. doi: 10.1148/rg.20181 80053

41. Lock G, Schmidt C, Helmich F, Stolle E, Dieckmann KP. Early experience with contrast-enhanced ultrasound in the diagnosis of testicular masses: a feasibility study. Urology. (2011) 77:1049-53. doi: 10.1016/j.urology.2010.12.035

42. Zequi Sde C, da Costa WH, Santana TB, Favaretto RL, Sacomani CA, Guimaraes GC. Bilateral testicular germ cell tumours: a systematic review. BJU Int. (2012) 110:1102-9. doi: 10.1111/j.1464-410X.2012.1 1056.x

43. Kachramanoglou C, Rafailidis V, Philippidou M, Bertolotto M, Huang DY, Deganello A, et al. Multiparametric sonography of hematologic malignancies of the testis: grayscale, color doppler, and contrast-enhanced ultrasound and strain elastographic appearances with histologic correlation. J Ultrasound Med. (2017) 36:409-20. doi: 10.7863/ultra.16.02013

44. Lock G, Schmidt C, Schröder C, Löning T, Dieckmann KP. Straight vessel pattern and rapid filling time: characteristic findings on contrast-enhanced sonography of testicular lymphoma. J Ultrasound Med. (2016) 35:1593-9. doi: 10.7863/ultra.15.05049

45. Engels M, Span PN, van Herwaarden AE, Sweep FCGJ, Stikkelbroeck NMML, Claahsen-van der Grinten HL. Testicular adrenal rest tumors: current insights on prevalence, characteristics, origin, and treatment. Endocr Rev. (2019) 40:973-87. doi: 10.1210/er.2018-00258

46. Patel SR, Richardson RL, Kvols L. Metastatic cancer to the testes: a report of 20 cases and review of the literature. J Urol. (1989) 142:1003-5. doi: 10.1016/S0022-5347(17)38969-3

47. Handa T, Nagai S, Hamada K, Ito I, Hoshino Y, Shigematsu M, et al. Sarcoidosis with bilateral epididymal and testicular lesions. Intern Med. (2003) 42:92-7. doi: 10.2169/internalmedicine.42.92

Conflict of Interest: The authors declare that the research was conducted in the absence of any commercial or financial relationships that could be construed as a potential conflict of interest.

Copyright (C) 2021 De Cinque, Corcioni, Rossi, Franceschelli, Colombo, Golfieri, Renzulli and Gaudiano. This is an open-access article distributed under the terms of the Creative Commons Attribution License (CC BY). The use, distribution or reproduction in other forums is permitted, provided the original author(s) and the copyright owner(s) are credited and that the original publication in this journal is cited, in accordance with accepted academic practice. No use, distribution or reproduction is permitted which does not comply with these terms. 